\title{
Predictions for Future Shopping Lists \& Coupons Using the Python Programming Language
}

AUTHORS: Mollie Bianchi, Joseph D'Ercole, Thomas Lawrynuik, Alexandra Leone

ScHOOL: Villanova Collehe

\section{Abstract}

In today's society Big Data is a commonly used marketing tool for companies to learn more about their customers. Given a large set of grocery store transaction data, we were to develop a means of using the data to benefit the customers and/or company. Big Data is a relatively new method of analysis and therefore not many high school students have had experience exploring data of this magnitude. We predicted future shopping lists and generated personalized coupons for each customer. To accomplish this we wrote a series of connected programs that calculate the average quantity and cost per food category. The averages were then used as the predicted amount for the next visit and individualized coupons were generated for the four most purchased categories. Due to time restraints and incomplete data sets, some of our hypotheses remain uninvestigated; however, more time and data would allow for these to be tested and confirmed. Overall, this program was created to enhance the customer's shopping experience and in return benefit the retailer.

\section{Key Words}

Big Data, Predicting, Python, Shopping Habits, Coupons

\section{Background}

As four students enrolled in the AP Program at Villanova College (VC) and members of the VC Programming Team, we are intrigued by the wide scope of applications that arise from Big Data Analysis. Our passion for intellectual exploration and skill development drives us; we approach the Big Data Challenge as an opportunity to exercise our creativity, common sense, curiosity, presentation skills, and programming expertise.

\section{Purpose}

Our goal for this challenge is to predict each customer's shopping list for the following week and through this experience, we aim to improve not only our programming skills but also develop our analytical mindset, and critical thinking skills. As a result of our exploration, we hope to be better prepared for success in post-secondary education and/or future careers in related computer science fields.

\section{Hypothesis}

If a program is created to compile data from a given set of statistics (e.g. spreadsheet) then a predicted shopping list for each customer can be generated.

For any given shopping trip:

If a person purchases a similar quantity of each product (e.g. 4, 3, 5 etc.), then the average quantity should be purchased on their next visit.

If a person purchases a growing quantity of each product (e.g. $4,5,6,7$, etc.), then they should buy more of that item on their next visit.

If a person purchases a diminishing quantity of each product (e.g. 7, 6, 5, 4, etc.), then they should buy less of that item on their next visit.

However, if a person usually buys the average amount and buys significantly less of a product on their most recent visit (e.g. usually buys 4 ; recently bought 1 ), then they should buy significantly more of that item on their subsequent trip. Likewise, if a person usually buys the average amount and then buys significantly more (e.g. usually buys 5 ; recently buys 10 ), then they should buy significantly less of that item during their next shop.

In addition, if a customer's shopping frequency is not consecutive (e.g. week 24, week 29), but then increases (e.g. week 29, week 32), then the amount they buy the subsequent visit should decrease. If a customer's shopping frequency is not consecutive (e.g. week 24, week 29), but then decreases (e.g. week 29, week $35)$, then the amount they buy the next visit should increase because they will need more items for the longer time period between shops. Furthermore, if multiple people buy the same item on the same week, then this should be the most common item purchased overall for that location. If multiple people do not buy an item on the same week, then this should be the least purchased item overall.

Presuming the aforementioned patterns are valid, if code is generated in Python to organize the data by customer number, then additional features predicting upcoming purchases and/or coupons will be generated. 


\section{Methodology}

Each time the program executes, the data is initially arranged by customer number. For a given customer, each distinct transaction's quantity and cost are added to running totals for the corresponding food category (ex. if the purchase is from the produce category, the running totals for produce quantity and produce monies are updated). Similarly, the total number of unique store visits for each customer is also compiled and stored for later use. From this, the customer's average quantity and average amount spent on each food category per visit are calculated. This average is a fair estimate of what a given customer will buy on their next visit to the store. Finally, a set of four $10 \%$-off coupons is created for each customer. Each set is composed of coupons for the customer's most frequently purchased items. If a customer has only ever bought from fewer than four unique food categories, the remaining coupons are automatically generated for one or more of the following food categories: seafood, dairy, food packaging, or produce.

\section{Results}

The program Functions2.1.py prints customer information in terms of predicted average amounts to be spent during subsequent visits to the Python shell. Functions2.2.py outputs to a file the expected shopping list for the individual customer's next shopping visit using the average amount purchased previously, as is mentioned in the Hypothesis section. Fig. 1.1, 1.2, and 1.3 showcase three individual customers predicted shopping lists based upon their average spending and number of items purchased for each category.

Functions2.3.py outputs to a file a customer's top 4 items as coupons, including their address and name information (i.e. postal code and customer number), with the "customer number + coupons" as the filename. Where a customer has purchased fewer than 4 items, automatic coupons are generated in the most popular categories (e.g. food packaging, dairy, etc.). Where a customer has purchased zero items, the prediction is that zero items will be purchased during the next visit, but four coupons are still generated (which is further explained in the Discussion section).

\section{Discussion}

While originally the team hoped to explore even more than predicting future shopping trips, the team was pleasantly surprised to accomplish so much without formal computer science
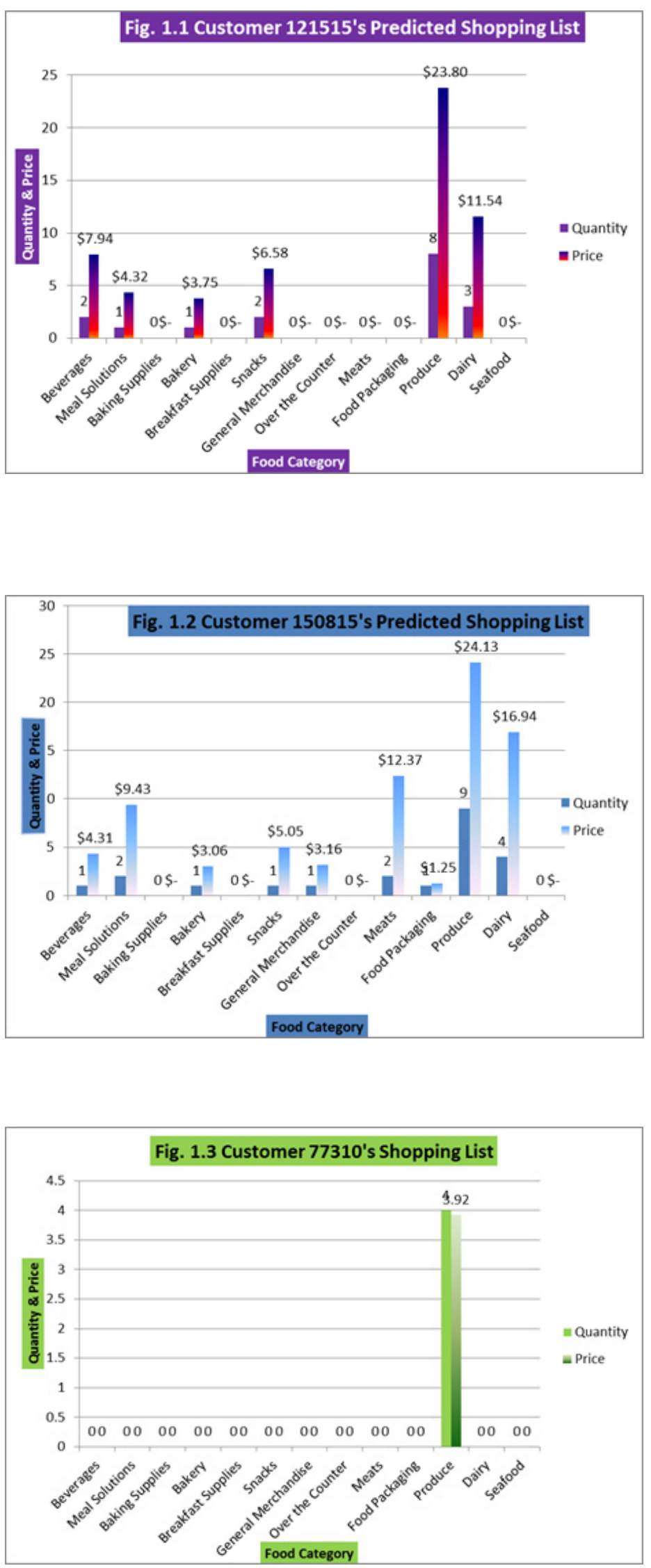
courses or training previous to this challenge. However, clearly there is more to do. With more time, further analyses would be conducted and algorithms created to account for customers with low purchase numbers and/or interesting/varied patterns of consumption between visits.

For example, during the production of the average algorithm, it was noted that there was an issue when a customer purchased zero items for a given category, in that there was a divide by zero error. This was accounted for by predicting that if zero items were purchased on all previous visits, then zero items would be purchased on the next visit as well. The team recognizes that this is not necessarily how predicting and buying habits work, but due to time constraints we wanted to a have a working prototype for the prediction program and coupon generator. With more time, the team would be interested in increasing the complexity of this part of the analysis.

Furthermore, the average algorithm does not take into account customers who buy an increasing number of items during each visit, nor does it address the fact that customers will not necessarily continue that trend to an infinite amount (in fact it is more likely they will at some point buy less of that given item). The same is true of decreasing trends. However, this could be added to the current series of programs to further increase the validity and accuracy of this coupon generator.

The coupon generator was not actually part of the team's original hypotheses and goals for the project, but it is a fruitful add-on. With more time, the team would be interested in exploring a coupon generator that produces coupons for the two most-bought items and two least-bought items for each customer (in addition to or in place of the other coupons already coded for).

Considerations were also given to weekly visit patterns (i.e. if customers were to adopt a weekly shopping pattern), and what the consecutive week's shopping lists would consist of in terms of products/categories and number of items for each (e.g. if a customer shopped week 25 , what would they buy week $26,27,28$, etc.). In terms of weekly patterns, the team thought it would be interesting to make a secondary program using the given data that predicts the week that is most popular for buying items from a given category and target a sale for that week (or group of weeks). For example, when do the most customers buy bread? Perhaps a sale on bread during that time period would be beneficial (or potentially raising the price during this time for greater profits).

Additionally, the team believes if more data were collected that indicated the specific brands rather than generic food categories (ex. how many high-end products were purchased), or streamed customers based on the type of grocery store (bargain, specialty, etc.) they shopped at, predictions of political preference would be possible. However, this is a basic version for analysis, and the team recognizes that it is a simplistic determining factor for such a complex topic. Yet it would be an excellent starting point.

This whole challenge has been an excellent experience of analyzing "big data". The entire VC Big Data Team looks forward to the next phase of this competition, and to the 2016 challenge as well.

\section{Appendix I Technical Methodology}

The provided Excel data was converted into multiple CSV files (one per relevant worksheet) and then opened using Microsoft Notepad. This conversion process provided opportunities to organize, manipulate, and utilize the data collection. Further to this, the files were saved as Python documents (BIGDATA.py, CUSTDATA.py, and STOREDATA.py) and the data was further modified into giant strings with one variable name per file, allowing the VC Team to create nested lists for use in coding prediction algorithms.

The original concept for predicting a customer's future shopping list was to create a series of dictionaries in Python. The benefit to this method focused upon using the customer number as a key to call up related data. However, upon experimentation, the team realized that this method was not only time-consuming, but also inefficient (both in terms of best coding practice and actual algorithmic procedure).

To solve this issue, the team re-approached the task using nested lists instead of dictionaries. The benefits of a nested list far outweighed that of a dictionary's key function, due to the streamlined coding syntax, ease of information access via indexing, and mutable nature of the list function itself. Nested lists were generated for each of the three data files, such that each cell of the outermost list contained a customer-specific list within it where applicable, and others contained related store data (i.e. the innermost list).

The program which generated these can be found in Functions2.0.py. The lists were saved to various files that were later accessed directly by Functions2.1.py, Functions2.2.py, and Functions 2.3.py. Functions2.0.py uses for loops to access the BIGDATA. py string and then by counting commas and using nested if statements, determines cells in the three lists, and prevents duplicates where applicable. This created the file OTHERLISTS.py which includes a list of category numbers (catlist), week numbers (weeklist) and customer numbers (custnumlist). 
Functions 2.0.py imports and utilizes BIGDATA.py (transaction data for each customer), CUSTDATA.py (personal information for each customer), STOREDATA.py (location information, etc. for each store), and the aforementioned OTHERLISTS.py.

Using BIGDATA.py (and then the same process for CUSTDATA. py), Functions2.0.py took each line of the string and converted it to a list (i.e. one list per transaction). Each of these lists was then inserted into an outermost list, such that each cell of the shell list contains a list containing individual transaction data. This overall nested list is named list2.

At this point the team created a new nested list called custav. This list compiles the total spending by category for each customer. Another list, custavwk, lists all weeks each customer visited a store, and was used to add the total weeks shopped during the data collection period to the custav list (i.e. append the custav list).

Functions2.0.py offered the ability to print the custav and custavwk lists to the Python shell. This output was copied and pasted to the CUSTDATA.py file to be called upon later in the team's coding process.

The team then began coding Functions2.1.py which requested user input in the form of an individual customer number, and then called upon the custav list data from CUSTDATA.py to generate the total number of items purchased, money spent, and transactions/ categories items were purchased from. This data was output to the shell for verification. Then the team coded a subprogram for calculating that customer's averages that was based upon the number of unique weeks customers visited a store, which in Functions2.1.py, prints to the Python shell only.

Functions2.2.py is strikingly similar to Functions2.1.py, except that the 2.2 version prints the individual customer's averages to a file (saved by customer number only), instead of the Python shell.
This intermediate program allowed for the team to practice file output and structural formatting. Leaping over 2.2, led the team to Functions2.3.py, in which the data is further analyzed and output as a coupon by customer number (e.g. file saved for customer number 1857 would be named '1857coupons'). When run, the 2.3 version creates a file saved in the same folder as Functions2.3.py, and the user must then open the file (specifying Word for formatting purposes) to view the four individualized coupons for the most purchased items. This program is the final product of the team's efforts, and showcases the possibility of predictive shopping lists and coupon generation.

\section{Appendix II Acknowledgements}

We would like to thank our mentor, Ms. Elissa Gelleny, for her support and aid throughout this process. Ms. Gelleny is a Senior Math/Science/Technology Teacher at Villanova College as well as the Moderator for VC's Computer Programming Team. While she provided proofreading services and/or commented on the Team's ideas upon request, Ms. Gelleny did not generate ideas for the Team or provide any technical programming or Python coding assistance. We thank Ms. Gelleny for her primary role as a facilitator.

Additionally, we would like to thank Dr. Sacha Noukovitch, along with the entire team of involved staff from SCINET and SAS alike, for providing us with both the opportunity to participate in the Big Data Challenge for High School Students and support/guidance throughout the journey. The quick response rate to our emailed questions from Dr. Noukovitch was greatly appreciated and instrumental in fostering our project's development.

\section{References}

1. Du, Rex Yuxing. "Measuring Contagion in the Diffusion of Consumer Packaged Goods." Journal of Marketing Research 48.1 (2011): $28-47$. Web.

2. Kohavi, Ron, Johannes Gehrke, William DuMouchel, and Joydeep Ghosh. KDD-2004: Proceedings of the Tenth ACM SIGKDD International Conference on Knowledge Discovery and Data Mining: August 22-25, 2004, Seattle, Washington, USA. New York, NY: ACM, 2004. Web.

3. "Don't Blame a Change in Shopping Habits, Britain's Supermarkets Just Got It so Wrong." The Telegraph. Telegraph Media Group, n.d. Web. 12 Feb. 2015.

4. "Grocery App Predicts Food Shopping Needs, NY I Stylus I Innovation Research \& Advisory." Stylus. N.p., n.d. Web. 12 Feb. 2015.

5. "Predictive Analytics Times." Predictive Analytics Times. N.p., n.d. Web. 12 Feb. 2015.

6. "Privacy Research Papers." Office of the Privacy Commissioner of Canada. N.p., n.d. Web. 12 Feb. 2015.

7. "Stat of the Day: $68 \%$ of Millennials Ask Friends Before Choosing a Restaurant." Advertising Age AdAgeStat RSS. N.p., n.d. Web. 12 Feb. 2015 


\section{REVIEW}

\section{by Jaime Pinto, \\ HPC Systems Administrator at SciNet and SOSCIP}

This is a good dissertation, in that the rationale for some decisions was explained, and in which the authors realize that research is indeed an organic and ever evolving process.

However, it is recommended that the authors be more precise in terminology. In the first paragraph of the Abstract, the expression "Big Data is a commonly used marketing tool ..." is ambiguous and may possibly be interpreted as incorrect, depending on the reader [1], and induce a unfavorable bias from the start.

It might be better worded as "In today's society Big Data analytics is a commonly used marketing tool for companies to learn more about their customers." [2] Or "Big Data is a relatively new method of analysis ..." taking the previous observation [1] into consideration, and what I consider to be the reason for ambiguity on the definition of Big Data [3].

[1] What Is Big Data? http://datascience.berkeley.edu/what-is-big-data/

[2] Big Data analytics? http://www.webopedia.com/TERM/B/big_data_analytics.html

[3] Is Big Data a Volume or a Technology? http://www.webopedia.com/TERM/B/big_data.html

As for the Purpose section: it is better to not mix the objective of the publication with that of the authors. Maybe segregate the two: one as the objective of this exercise for the students, primarily as a comment or a by-product, (but the purpose be focused on the predicted shopping list for the customers.)

In the Methodology section the concept of dollar amount is introduced for the $10 \%$-off coupons, and that was never described as one of the purposes for the publication, although it was briefly mentioned in the abstract. In addition, the Hypothesis only makes references to quantity (units) of products per visit, with no reference to prices, so the coupons as a possible outcome here is a complete surprise.

Instead, I expected to see a little more detail on how the data was processed and the results generated (not necessarily how the python code was implemented), rather than how the results would be used, which is something that could, in turn, go into the Purpose section.

In Appendix 1 the authors provide a good description of the functionality and capability of each piece of code used.

For the next phase of the project it is recommended to test the hypothesis through predictive exercises that could be built as follows :

For the next phase of the project it is recommended to test the hypothesis through predictive exercises that could be built as following:

If you have a sequential collection of historical data points, for instance, $1,2,3, \ldots, 10$ and they are assumed to be intrinsically predictive in nature, you can use sequence $1,2,3$ and try to predict what 4asFunctionOf $(1,2,3)$ will be and measure the difference from the actual 4. Then calculate 5 asFunction $f(2,3,4), 6$ asFunctionOf $(3,4,5)$ and so on. The smaller and more consistent the collection of all those differences, the better the predictive model will be. You can further refine the model by adding more history points for each pass, such as 5asFunctionOf(1,2,3,4) and 6asFunctionOf(2,3,4,5), ... and so on.

The ultimate test of how good the model is would be to predict the "real future", such as 11 asFunctionOf(10,9,8,7), and end up with a minimum acceptable error. 